\title{
Singular Soliton to the Hyperbolic Generalization of the Burgers Model
}

\author{
Haci Mehmet Baskonus ${ }^{1}$, Faruk Düşünceli ${ }^{2}$ \\ 1. Department Computer Engineering, University of Munzur, TURKEY, Tunceli, \\ 2. Faculty of Architecture and Engineering, Mardin Artuklu University, Mardin, Turkey \\ hmbaskonus@gmail.com, farukdusunceli@artuklu.edu.tr
}

\begin{abstract}
In this paper, new singular soliton solution is found to the hyperbolic generalization of the Burgers equation. 2D and 3D graphs are also presented. At the end of paper, a conclusion is introduced as well by mentioning novel aspects of paper.
\end{abstract}

Keywords - Exponential function method, Hyperbolic Generalization of the Burgers equation, Singular soliton solution.

\section{Introduction}

The models arising in wave propagation are mostly expressed in time domain [1-4]. Such models also introduces the shapes of waves from time to time. One of such model is hyperbolic generalized Burgers equation (GBE) defined by [5-7]

$$
\tau u_{t t}+u_{t}+u u_{x}+B u_{x}-\kappa u_{x x}-\lambda u(u-s)(u+q)=0,
$$

where $\tau, B, \kappa, \lambda, s, q$ are arbitrary real constants.

\section{General Properties of EFM}

General and more deeper propertiess of EMF have been presented by varios scientist to the literature in $[8,9]$.

\section{Implementation of the EFM}

Applying $u(x, t)=U(\xi), \xi=k x-c t$ into Eq.(1), we get following nonlinear ordinary differential equation

$$
\left(\tau c^{2}-\kappa k^{2}\right) U^{\prime \prime}+(k B-c) U^{\prime}+k U U^{\prime}-\lambda U^{3}+(s \lambda-q \lambda) U^{2}+s \lambda q U=0,
$$

Considerin balance principle, the value of $N$ can be found as

$$
N=1 \text {. (3) }
$$

Then, we can write following equations;

$$
\begin{gathered}
U=A_{0}+A_{1} \exp (-\Omega(\xi)),(4) \\
U^{\prime}=A_{1} \exp (-\Omega(\xi)) \Omega^{\prime},(5) \\
U^{\prime \prime}=\cdots(6)
\end{gathered}
$$

where $A_{1} \neq 0$. Using Eqs. $(4,5,6)$ into Eq. $(2)$, we get an algebraic system of equations. Solving this system of equations, we reach following coefficients

$$
A_{0}=\frac{1}{2}\left(-q+w A_{1}\right), s=-\frac{2 c-2 B k+k q+q \lambda A_{1}}{2 \lambda A_{1}}, \tau=\frac{2 k^{2} \kappa+\left(k+\lambda A_{1}\right) A_{1}}{2 c^{2}}, \mu=\frac{1}{4}\left(w^{2}-\frac{q^{2}}{A_{1}^{2}}\right) .
$$

Substituting these coefficients into Eq.(4), we find the following singular soliton solution as

$$
u(x, t)=\frac{-q+w A_{1}}{2}+\frac{\left(q^{2}-w^{2} A_{1}^{2}\right) \operatorname{coth}(k \varpi x-\varpi c t+\varpi E)}{2 q+2 w A_{1} \operatorname{coth}(k \varpi x-\varpi c t+\varpi E)}, \text { (7) }
$$

where $q^{2} / A_{1}^{2}>0$ and $\varpi=q / 2 A_{1}$ for validity condition of Eq.(7). 

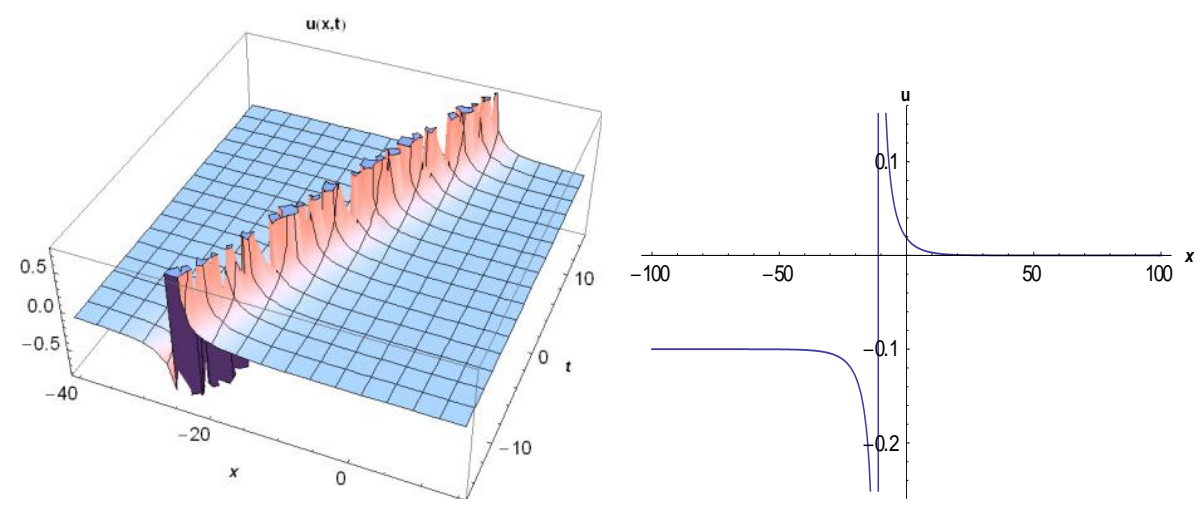

Fig.1 The 3D and 2D surfaces of Eq.(7)

\section{Conclusion}

In this paper, we have applied EFM to the GBE. singular soliton solution has also obtained. It can be observed that this solution has satisfied to the GBE via various computational programs. For better understanding of physical meanings of results, 2D and 3D surfaces have also plotted. Therefore, it can be said that this method is very powerfull and can be applied other nonlinear with high nonlinearity.

\section{References}

[1] F.Dusunceli, E.Celik, "Numerical solution for high-order linear complex differential equations with variable coefficients", Numerical Methods for Partial Differential Equations, 2017, 34(5), 1645-1658.

[2] A.Ciancio, V.Ciancio, F.Farsaci, "Wave propagation in media obeying a thermo viscoan elastic model”, U.P.B. Scientific Bullettin University Politehnica of Bucharest Series A: Applied Mathematics and Physics, 2017, 69(4), 69 -79.

[3] C.Cattani, A.Ciancio, "On the fractal distribution of primes and prime-indexed primes by the binary image analysis", Physica A, 2016, 460, 222-229.

[4] F.Dusunceli, E.Celik, “An Effective Tool: Numerical Solutions by Legendre Polynomials for High-Order Linear Complex Differential Equations, British Journal of Applied Science and Technology, 2015, 8(4), 348-355.

[5]V.A.Vladimirov, C. Maczka, "Exact Solutions of Generalized Burgers Equation Describing Travelling Fronts and Their Interaction", Reports on Mathematical Physics, 2007, 60(2), 317-328.

[6] A.S. Makarenko, M.N. Moskalkov, S.P. Levkov, "On blow-up solutions in turbulence", Physics Letters A, 1997, 235(4), 391-397.

[7] A. S. Makarenko, "New differential equation model for hydrodynamics with memory effects", Reports on Mathematical Physics, 2000, 46, 183-190.

[8] M.G. Hafez, M.N. Alam, M.A. Akbar, Travelling wave solutions for some important coupled nonlinear physical models via the coupled Higgs equation and the Maccari system, Journal of King Saud University - Science, 27, 105-112, 2015.

[9] Uddin S., Alam N., Hossain S.M.S., Samiu H. and Akbar M.A., Some New Exact Traveling Wave Solutions to the (3+1)-Dimensional Zakharov-Kuznetsov Equation and the Burgers Equations via $\operatorname{Exp}(-\phi)$-Expansion Method, Frontiers of Mathematics and Its Applications, 1(1), 1-8, 2014. 